\title{
Analyst
}

\section{Mid-infrared fiberoptic evanescent field spectroscopy for in- situ monitoring of tetrahydrofuran hydrate formation and dissociation}

\begin{tabular}{|r|l|}
\hline Journal: & Analyst \\
\hline Manuscript ID & AN-COM-10-2016-002237.R1 \\
\hline Article Type: & Communication \\
\hline Date Submitted by the Author: & n/a \\
\hline Complete List of Authors: & $\begin{array}{l}\text { Mizaikoff, Boris; Institut für Analytische und Bioanalytische Chemie, } \\
\text { University of Ulm } \\
\text { Schwenk, Matthias; Institut für Analytische und Bioanalytische Chemie, } \\
\text { University of Ulm } \\
\text { Katzir, Abraham; Tel Aviv University, The School of Physics and Astronomy }\end{array}$ \\
\hline
\end{tabular}




\section{Journal Name}

Received 00th January 20xx,

Accepted 00th January 20xx

DOI: $10.1039 / x 0 x \times 00000 x$

www.rsc.org/

Tetrahydrofuran is a relevant auxiliary molecule when storing carbon dioxide or hydrocarbons as gas hydrates. The present study demonstrates the applicaton of in-situ mid-infrared fiberoptic evanescent field absorption spectroscopy for studying the formation and dissociation of THF hydrates. Thereby, the utility of this analytical technique providing unique molecularlevel insight even at harsh environmental conditions is evidenced.

Gas hydrates - or more general, clathrate hydrates - are crystalline host-guest compounds, which are able to trap small molecules inside a network of hydrogen-bonded water structures. The guest size depends on the cavity diameter and thereby determines the final structure of the clathrate hydrate. In the case of natural gas hydrates, the three main structures are the cubic structures $\mathrm{sl}$ and sll, and the hexagonal structure $\mathrm{sH}$. Each structure is composed of a different number of water molecules combined into the respective water cages with an increasing capability to trap larger molecules from $\mathrm{sl}$ to $\mathrm{sH} .{ }^{1,2}$ The formation conditions are generally in the range of several $\mathrm{MPa}$ pressure, and at temperatures close to the freezing point of water with the respective values being interrelated. Detailed tabulated phase equilibria data for many systems can be found in literature. ${ }^{3}$ Next to gaseous guests such as short chained hydrocarbons (e.g., methane, ethane, propane), carbon dioxide, and nitrogen, also small ethers including tetrahydrofuran (THF) may form hydrates at appropriate conditions. In the case of gaseous clathrate hydrates, the diffusion of gas into the liquid phase, adequate pressure, and sufficient supply of the guest are among the key requirements for hydrate growth. Tetrahydrofuran hydrates on the contrary form directly from liquid mixtures of water and THF at an ideal molar ratio of 1:17 THF:water ${ }^{4}$ at atmospheric pressure and at temperatures below 277.4 K. ${ }^{5}$ Due to these modest formation conditions,

\footnotetext{
a. Institute of Analytical and Bioanalytical Chemistry, Ulm University, Albert Einstein-Allee 11,Ulm 89081, Germany.E-mail: boris.mizaikoff@uni-ulm.de

${ }^{b .}$ The School of Physics and Astronomy, Tel Aviv University, Tel Aviv 69978, Israel
}

the experimental effort when studying THF hydrates is low compared to e.g., $\mathrm{CO}_{2}$ or hydrocarbon hydrates, as THF hydrates can be synthesized in virtually any kind of reaction vessel connected to a cooling system. In contrast, gas containing clathrates require a high-pressure autoclave along with appropriate gas supply/mixing systems. The resulting structure of pure THF hydrates is known as sll, which comprises 16 dodecahedral $\left(5^{12}\right)$ and 8 hexakaidecahedral $\left(5^{12} 6^{4}\right)$ cages. The larger hexakaidecahedral cavities are occupied by THF, while the smaller dodecahedral cavities remain empty. ${ }^{2,4,6}$

Next to the capability forming hydrates of its own, THF also has proven to be an excellent thermodynamic promoter during the formation of carbon dioxide and methane gas hydrates. The addition of small amounts of THF actively shifts the formation conditions towards more modest regimes, thereby resulting in lower pressure and higher temperature requirements. ${ }^{7-12}$ The utilisation of THF as an assistive molecule for storing hydrogen as hydrate is another interesting concept that is currently being researched. ${ }^{13-16}$ These properties render THF hydrates an appealing model system to investigate fundamental processes involved during hydrate formation and dissociation in molecular-level detail for further application as auxiliary molecule in hydrate storage scenarios involving various gases.

In the field of hydrate research, Raman spectroscopy is usually considered the method of choice for monitoring hydrate formation. Accordingly, Prasad et al. investigated THF hydrate across a sizeable temperature range (i.e., $90-300 \mathrm{~K}$ ) via Raman spectroscopy, and observed a splitting of the ring breathing mode at $90 \mathrm{~K}$, which may be attributed to different guest sites within structure sll hydrate. ${ }^{17}$ Infrared (IR) spectroscopic techniques are rarely used, which is mainly attributed to the rather strong absorption of water in the midinfrared (MIR; 3-15 $\mu \mathrm{m}$ ) regime usually rendering the detection of additional species difficult. Bertie and Othen investigated for the first time ethylene oxide clathrate hydrate in the range of $20-4000 \mathrm{~cm}^{-1}{ }^{18,19}$ Fleyfel and Devlin later applied IR spectroscopy when studying carbon dioxide and THF hydrates 
determining pronounced bands for $\mathrm{CO}_{2}$ in the small and large cages depending on the present structure, as well as a band characteristic for THF in the large cages of structure sll. ${ }^{20}$ More recently, several publications have demonstrated the fundamental potential of IR spectroscopy in the field of hydrate science. ${ }^{21-25}$ The application of fiberoptic evanescent field absorption spectroscopy (MIR-FEFS) was pioneered by the research team of Mizaikoff introducing significant advancements for MIR-based monitoring systems in gas hydrate research. The developed gas hydrate monitoring systems enable performing in-situ dynamic IR studies during hydrate formation and dissociation, since fiberoptic waveguides may be subjected to elevated pressure conditions. Consequently, methane, ethane and propane containing hydrates have been investigated via MIR-FEFS, as reported by Dobbs et al. and Luzinova et al. ${ }^{26,27}$ Recently, this technique was adapted towards the investigation of carbon dioxide containing gas hydrates, and for monitoring relevant additives during hydrate formation and dissociation. ${ }^{28}$

The present study demonstrates the first application of MIRFEFS for in-situ monitoring of the formation and dissociation processes of THF hydrates. Spectroscopic information at the molecular-level of the relevant reactants (i.e., water and THF) was correlated with continuously recorded temperature traces, thereby introducing MIR-FEFS as an innovative analytical strategy for studying THF hydrates. The developed reaction vessel enables the IR spectroscopic investigation of the initial reaction mixture (i.e., water/THF molar ratio 17:1), and its in-situ conversion into crystalline hydrate. MIR-FEFS is based on the principles of attenuated total reflection (ATR) spectroscopy, i.e., more general, internal reflection spectroscopy (IRS) introduced by Harrick. ${ }^{29}$ While in conventional IR-ATR usually hemispherical single-reflection or trapezoidal multi-reflection crystalline waveguides (e.g., made from $\mathrm{ZnSe}, \mathrm{ZnS}, \mathrm{Ge}, \mathrm{Si}$, diamond, etc.) are applied, MIR-FEFS utilizes cylindrically shaped MIR-transparent optical fibers, here, polycrystalline fiberoptics made from $\mathrm{AgCl}_{0.3} \mathrm{Br}_{0.7}$ as IRS

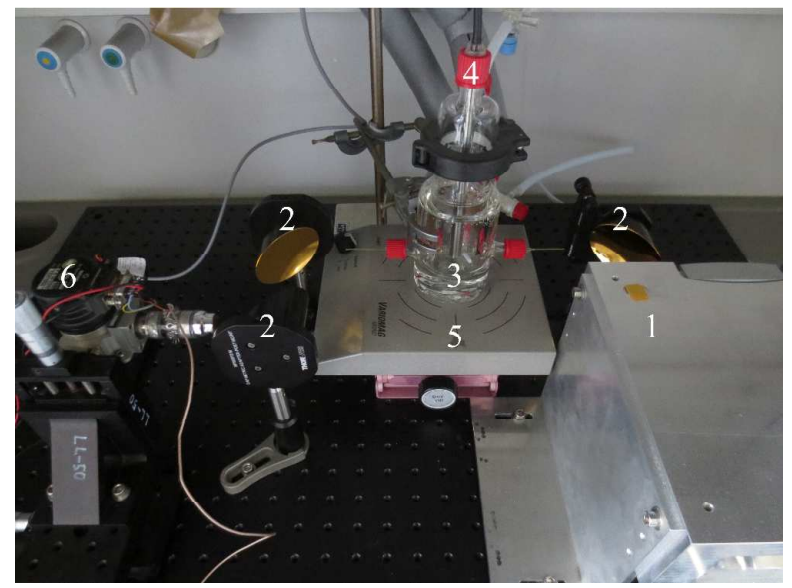

Fig. 1 MIR-FEFS setup: (1) Bruker Alpha FTIR spectrometer, (2) gold coated off-axis parabolic mirrors (OAPMs), (3) customized glass reaction vessel, (4) Pt100 thermistor, (5) magnetic stirrer, (6) Stirling-cooled mercury-cadmium-telluride (MCT) detector. waveguide. Light is propagated via total internal reflection along the fiber giving rise to an evanescent field at each reflection point, which exponentially decays in intensity into the adjacent medium. Thereby, the detection of IR-active species in the vicinity of the fiber surface is enabled via evanescent field absorption spectroscopy. ${ }^{30}$

The experimental setup (Fig. 1) is assembled inside a darkened fume hood to minimize light-induced degradation of the fiber. A custom designed glass reaction vessel (Fig. 1 \#3) featured fiberoptic feed-through ports sealing the silver halide fiber (diameter $900 \mu \mathrm{m}, \mathrm{AgCl}_{0.3} \mathrm{Br}_{0.7}$ ) into the vessel, thus enabling spectroscopic access. Silver halide fibers are mechanically robust while retaining some flexibility due to their polycrystallinity, and feature a wide spectral window in the MIR region. ${ }^{30}$ The reaction vessel has been constructed double-walled facilitating effective cooling of the reaction solution via a Huber Ministat 125 cooling system. A Pt100 thermistor (Fig. 1 \#4) was immersed into the solution just above the fiber surface for recording the process temperature throughout the entire experiment close to the analytical volume probed by the evanescent field. A Bruker Alpha FTIR spectrometer (Bruker Optik GmbH, Ettlingen, Germany) (Fig.1 $\# 1$ ) in an OEM version was used. Three 2" gold-coated off-axis parabolic mirrors (OAPMs; Edmund Optics Inc., Barrington, NJ, USA) (Fig. 1 \#2) guided the IR radiation from the source via the fiberoptic towards the detector element of an external Stirlingcooled mercury-cadmium-telluride (MCT) detector (Model K508, Infrared Associates, Stuart, FL, USA) (Fig.1 \#6). The detector was connected to the FTIR spectrometer via an impedance-matched MCT-1000 pre-amplifier. Underneath the reaction vessel, a magnetic stirrer (Thermo Fisher Scientific Inc., Waltham, MA, USA) (Fig. 1 \#5) has been mounted. The experiments were conducted in a solution of demineralized water and THF (Merck KGaA, Darmstadt, Germany with a purity of $\geq 99.9 \%$ ) at a molar ratio of $17: 1$. The solution was stirred for approx. $1 \mathrm{~h}$ prior to analysis enabling adequate mixing of both liquids. Prior to recording a background spectrum of air, the fume hood comprising the setup was flushed with dry compressed air for $1.5 \mathrm{~h}$. Afterwards, the solution was added to the reaction vessel. The experiment was not started before another $1.5 \mathrm{~h}$ ensuring equilibration of the entire environment. The cooling system was programmed such that the solution was cooled to approx. $1{ }^{\circ} \mathrm{C}$ during $1 \mathrm{~h}$ after reaching an initial temperature of approx. $22^{\circ} \mathrm{C}$ during $10 \mathrm{~min}$. Initial fluctuations were resulting from equilibrating the cooling system. Upon reaching the target temperature $\left(1^{\circ} \mathrm{C}\right)$, it was maintained constant for $72 \mathrm{~h}$ following heating to $22{ }^{\circ} \mathrm{C}$ during $1 \mathrm{~h}$. IR spectra were recorded at intervals of $10 \mathrm{~min}$ with a spectral resolution of $2 \mathrm{~cm}^{-1}$ between $4000-600 \mathrm{~cm}^{-1}$. Each spectrum averaged 100 spectral scans. The reaction solution was stirred throughout the entire measurement. Eventually, the stirrer stopped due to the formation of crystalline hydrate. 
Fig. 2 shows exemplary MIR-FEFS spectra (i.e., solution vs. solid phase) obtained during a hydrate formation experiment. The black spectrum shows the spectral features of the reaction solution at the start of the experiment, whereas the red spectrum reveals the characteristics of THF hydrate obtained later during the experiment. Water has several pronounced absorption bands in the MIR region, i.e., the $\mathrm{OH}$ stretch vibration $\left(v_{\mathrm{OH}}\right)$ located at $3750-2750 \mathrm{~cm}^{-1}$, the $3^{\text {rd }}$ overtone of the libration mode $\left(3 v_{\mathrm{L}}\right)$ at $2300-1900 \mathrm{~cm}^{-1}$, the $\mathrm{HOH}$ bending vibration $\left(v_{2}\right)$ at 1850 and $1520 \mathrm{~cm}^{-1}$, and the libration mode $\left(v_{\mathrm{L}}\right)$ located below $1000 \mathrm{~cm}^{-1}$. Each of these features is specifically altered upon the crystallisation of hydrates, as

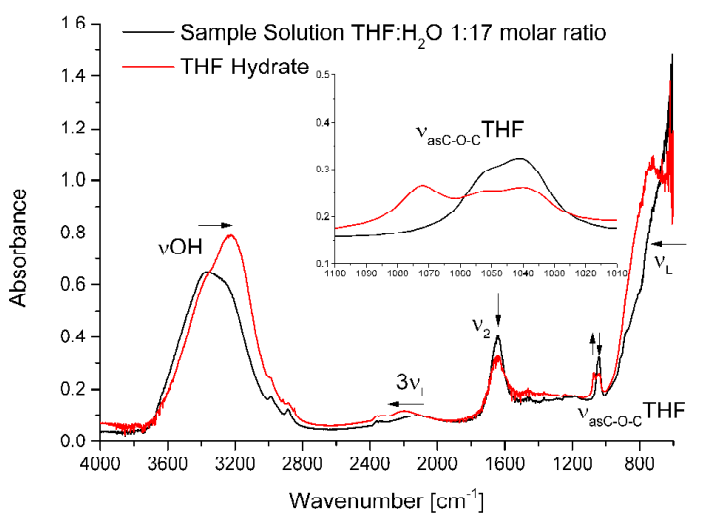

Fig. 2 Exemplary MIR-FEFS spectra recorded during a THF hydrate experiment; black: spectrum of the sample solution at the start of the experiment; red: spectrum with THF hydrate present. Inset: $v_{\text {asc-o-c }}$ of THF appearing with a shoulder at approx. $1051 \mathrm{~cm}^{-1}$.

already shown for methane, ethane, propane, and carbon dioxide containing hydrates via MIR-FEFS. ${ }^{26-28}$ The respective changes are similar to those appearing during cooling or crystallisation of water. ${ }^{31,32}$ The resulting structure of THF hydrate is cubic sll; hence, the spectroscopic changes during this study are expected to be qualitatively similar to previously conducted experiments with sll mixed $\mathrm{CO}_{2} / \mathrm{THF}$ hydrate. ${ }^{28}$ As can be derived from Fig. 2, the $v_{\mathrm{OH}}$ shifts to the red, while the $3 v_{\mathrm{L}}$ and the $v_{\mathrm{L}}$ feature shift to the blue. Furthermore, the $v_{2}$ decreases in intensity upon hydrate formation.

Next to water, THF also reveals distinctive absorption features, which are evident in the region of approx. $3015-2830 \mathrm{~cm}^{-1}$, and as a feature centered at $1041 \mathrm{~cm}^{-1}$. These spectral features are attributed to the $\mathrm{CH}$ stretching vibrations $\left(v_{\mathrm{CH}}\right)$, and the asymmetric $\mathrm{C}-\mathrm{O}-\mathrm{C}$ stretch vibration ( $\left.v_{\text {asc-o-c }} \mathrm{THF}\right)$, respectively (see Fig. 2). ${ }^{33}$ The $v_{\text {asc-o-c }}$ of THF appears with a shoulder at approx. $1051 \mathrm{~cm}^{-1}$, as magnified in the inset of Fig. 2. After comparison of this feature with literature it is apparently resulting from a mixture of two different species. Based on the findings of Mizuno et al. and regarding the molar fraction of water in the reaction solution (i.e., in this study 0.94), the detected THF feature may be interpreted as a mixture of THF connected to two $\left(1053 \mathrm{~cm}^{-1}\right)$ or at least three water molecules $\left(1038 \mathrm{~cm}^{-1}\right){ }^{33}$ The inset of Fig. 2 provides a detailed view of the region of interest regarding the $v_{\text {asc-o-c }}$ of THF. In the hydrate spectrum (red), a new feature centered at 1072 $\mathrm{cm}^{-1}$ was observed next to the broad feature of THF in aqueous solution (@ approx. $1051 / 1040 \mathrm{~cm}^{-1}$ ). This feature has been assigned by others to the asymmetric $\mathrm{C}-\mathrm{O}-\mathrm{C}$ stretch vibration of THF inside the large $5^{12} 6^{4}$ cages of the resulting sll hydrate structure. ${ }^{20,23,24,34}$ The fact that for THF in solution there is still a signal evident may be explained by remaining non-converted
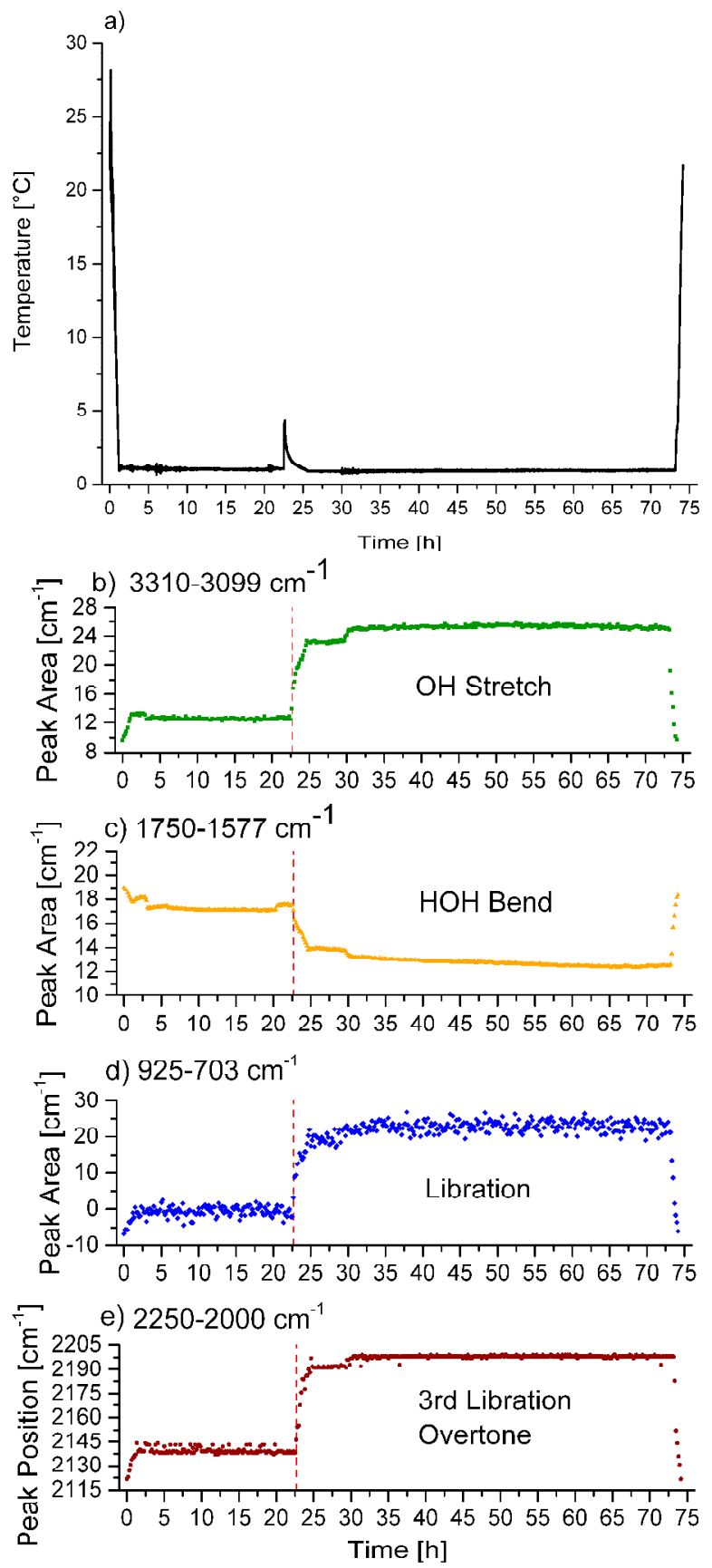

Fig. 3 Temperature trace (a) and spectral data traces of water absorption features with time during a hydrate formation experiment $(b-e)$; red dashed lines indicate the time of nucleation: approx. $22.7 \mathrm{~h}$. 
water/THF in the vicinity of the fiber after initiation of crystal formation. Changes of these features with time were monitored via peak integration and peak picking methods of individual characteristic features.

Fig. 3 displays the evolution of the absorption features of water throughout a hydrate formation experiment. Next to the spectroscopic data traces, the temperature trace is displayed clearly revealing the initiation of nucleation, as the temperature increases to approx. $4.32{ }^{\circ} \mathrm{C}$ after approx. $22.7 \mathrm{~h}$ into the experiment. In order to compensate interferences of water vapour mainly in the region of the $\mathrm{HOH}$ bending absorption (Fig. 2), a compensation algorithm provided by the spectrometer software (Bruker OPUS software package) was applied to the spectra prior to integration. It is clearly evident that simultaneous with hydrate nucleation the spectral data shift sharply (indicated by red dashed line in Fig. 3 b-e) reaching an equilibrated state with no more changes detectable. Only upon heating of the sample solution to approx. $22{ }^{\circ} \mathrm{C}$ and thereby dissociating the hydrate these shifts recover to the state prior to nucleation (starting after approx. $73.3 \mathrm{~h}$ ). These sharp shifts occurring simultaneously to the temperature peak indicate that nucleation occurs in immediate vicinity of the silver halide fiber surface. This direct response renders MIR-FEFS a novel and highly promising analytical technique for studying hydrate formation, and specifically THF hydrates at molecular level detail and in real time. In the following, the spectral changes associated with water are discussed in detail. The $\mathrm{v}_{\mathrm{OH}}$ feature shifts to the red (Fig. 2), which is interpreted as an increase of highly networked water species in the hydrate phase. ${ }^{31}$ Quantitative monitoring of this change was obtained via peak area integration (3310-3099 $\mathrm{cm}^{-1}$; Fig. 3 b). ${ }^{35}$ For analysis of the $v_{2}$ feature, the area of $1750-1577 \mathrm{~cm}^{-1}$ was integrated. The resulting decreases in intensity (Fig. 2), and as a consequence, the decrease in peak area (Fig. $3 \mathrm{c}$ ) upon hydrate formation is likely due to a loss of induced transition dipole moment with increasing solidification of the sample. ${ }^{31}$ Upon hydrate formation, the $v_{L}$ shifts to the blue (Fig. 2), thereby emerging within the detectable spectral region of the present setup limited by the spectral bandwidth of the Stirling-cooled MCT detector system. Analogous to the $\mathrm{v}_{\mathrm{OH}}$ absorption, partial peak area integration has been applied to the $v_{\mathrm{L}}$ at $925-703 \mathrm{~cm}^{-1}$ (Fig. $3 \mathrm{~d}$ ), which enables monitoring of the shift towards higher wavenumbers, even though the feature appears not fully evident due to the limited detectable spectral range. While these features have been analysed via peak area integration methods, a peak picking method has been utilized to monitor the blue shift of the $3 v_{\mathrm{L}}$ feature. Prior to peak picking, the wavelength segment of $2250-2000 \mathrm{~cm}^{-1}$ has been smoothed via a moving average method (software package Essential FTIR 3.50.047; Operant LLC, Madison, WI, USA). The resulting peak positions vs. time are displayed in Fig. 3 e. Likewise, a sharp shift is observable at the point of nucleation, which is in excellent agreement with the other monitored features.

To monitor changes associated with THF with time, the respective spectral features of THF have likewise been integrated. Integration borders were set at $1061-1020 \mathrm{~cm}^{-1}$ for the $v_{\text {asc-o-c }}$ of THF in aqueous solution (Fig. 4, black dataset), and at $1088-1061 \mathrm{~cm}^{-1}$ for the $v_{\text {asc-o-c }}$ of THF in the hydrate structure (Fig. 4; red dataset).

Similar to the water features displayed in Fig. 3, the changes related to the $v_{\text {asc-O-c }}$ feature of THF occur simultaneously with hydrate nucleation at 22.7 hours (Fig. 3 a). A sharp decrease in peak area is evident for the $v_{\text {asc-o-c }}$ feature of THF in solution (Fig. 4, black dataset), while an increase appears for the feature assigned to the hydrated species following this point of time (Fig. 4, red dataset).

Hence, this study provides unique continuously monitored

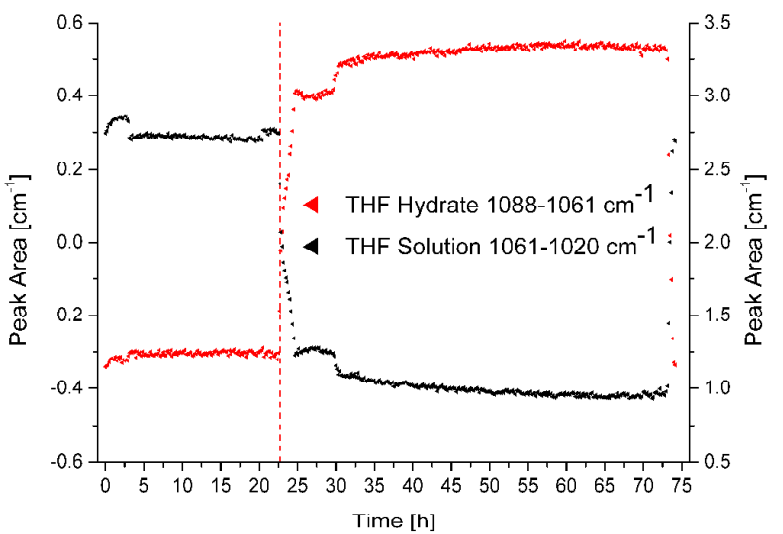

Fig. 4 Peak area integration evaluating the evolution of $v_{\text {asc- }}$ o-c of THF vs. time. The feature originating from THF in aqueous solution (black, right ordinate) decreases sharply at the point of nucleation (i.e., @ 22.7 hours, red dashed line), while the feature assigned to THF inside the clathrate structure behaves complementarily and increases sharply (red, left ordinate).

spectral evidence that indeed dissolved THF is continuously enclathrated into hydrate ice cages, as the associated spectral features behave complementarily until equilibrium is reached. At equilibrium, hydrate formation is assumed to be completed. Upon dissociation of the THF hydrate by heating it was proven that both features recover their initial state. The incorporation of THF molecules into the hydrate structure results in a depletion of THF in the remaining solution, which is clearly evidenced in the present experiments. The observed sharp changes occurring simultaneously in both datasets display the incorporation of THF molecules into the clathrate structure in real time onwards after $22.7 \mathrm{~h}$ into the experiment. In order to substantiate these findings, this experiment has been repeated individually three times with all experiments revealing nucleation simultaneous with sharp changes of the monitored spectral features of water and THF. It should be noted that in comparison to previous reports (see refs. ${ }^{20,23,24,34}$ ) identifying the feature at $1072 \mathrm{~cm}^{-1}$ as the signal of the $v_{\text {asc-o-c }}$ THF feature inside the large hexakaidecahedral sll cage, this feature was evident already at significantly higher temperatures during the present experiments. 


\section{Conclusions}

This study demonstrates for the first time the utility of infrared fiberoptic evanescent field absorption spectroscopy for in-situ monitoring the formation and dissociation of THF hydrates in real time and at molecular level detail. During THF hydrate formation the spectral changes associated with water and THF could be unambiguously identified, and correlated to the recorded temperature traces. Unique spectroscopic access is facilitated via a silver halide fiber submerged into the reaction solution serving as attenuated total reflection waveguide. Thereby, direct studies of hydrates providing molecular-level insight on the changes occurring during clathrate formation and dissociation for THF-containing hydrates is available for the first time. The present study reveals the potential of MIRFEFS-based monitoring systems not only for in-situ studies of hydrates, but corroborates the utility of fiberoptic IR-ATR spectroscopy in demanding analytical environments by pairing direct spectroscopic access with mechanical robustness.

\section{Acknowledgements}

The authors greatly acknowledge the collaboration with A. Katzir and his research team at Tel-Aviv University (Israel) for providing customized silver halide fibers. Furthermore, the machine shop at UIm University is acknowledged for assistance in establishing the MIR-FEFS gas hydrate setup. Partial financial support of this work by the European Union FP7 Project SCHeMA - Integrated in Situ Chemical Mapping Probes (Grant Agreement Number 614002) is gratefully acknowledged.

\section{Notes and references}

1 E. D. Sloan and C. A. Koh, Clathrate Hydrates of Natural Gases, CRC Press, Boca Raton, 3rd edn., 2008, ch. 2, 45.

2 E. D. Sloan and C. A. Koh, Clathrate Hydrates of Natural Gases, CRC Press, Boca Raton, 3rd edn., 2008, ch. 2, 55.

3 E. D. Sloan and C. A. Koh, Clathrate Hydrates of Natural Gases, CRC Press, Boca Raton, 3rd edn., 2008, ch. 2, pp. 358-535.

4 S. R. Gough and D. W. Davidson, Can. J. Chem., 1971, 49, 26912699.

5 S. Devarakonda, A. Groysman and A. S. Myerson, J. Cryst. Growth, 1999, 204, 525-538.

6 D. W. Davidson, Water - A Comprehensive Treatise, Plenum Press, New York, 1973, vol. 2, ch. 3, pp. 115-234.

7 J.-P. Torré, D. Haillot, S. Rigal, R. de Souza Lima, D. C. and B. J.-P., Chem. Eng. Sci., 2015, 126, 688-697.
8 J.-P. Torré, M. Ricaurte, C. Dicharry and D. Broseta, Chem. Eng. Sci., 2012, 82, 1-13.

9 J.-P. Torré, C. Dicharry, M. Ricaurte, D. Daniel-David and D. Broseta, Energy Procedia, 2011, 4, 621-628.

10 H. P. Veluswamy, S. Kumar, R. Kumar, P. Rangsunvigit and P. Linga, Fuel, 2016, 182, 907-919.

11 H. P. Veluswamy, A. J. H. Wong, P. Babu, R. Kumar, S. Kulprathipanja, P. Rangsunvigit and P. Linga, Chem. Eng. J., 2016, 290, 161-173.

12 A. Delahaye, L. Fournaison, S. Marinhas, I. Chatti, J.-P. Petitet, D. Dalmazzone and W. Fürst, Ind. Eng. Chem. Res., 2006, 45, 391397.

13 L. J. Florusse, C. J. Peters, J. Schoonman, K. C. Hester, C. A. Koh, S. F. Dec, K. N. Marsh and E. D. Sloan, Science, 2004, 306, 469471.

14 H. Lee, J. Lee, D. Y. Kim, J. Park, Y. Seo, H. Zeng, I. L. Moudrakovski, C. I. Ratcliffe and J. A. Ripmeester, Lett. Nat., 2005, 434, 743-746.

15 T. A. Strobel, C. J. Taylor, K. C. Hester, S. F. Dec, C. A. Koh, K. T. Miller and E. D. Sloan, J. Phys. Chem. B, 2006, 110, 17121-17125.

16 T. Sugahara, J. C. Haag, P. S. R. Prasad, A. A. Warntjes, E. D. Sloan, A. K. Sum and C. A. Koh, J. Am. Chem. Soc., 2009, 131, 14616-14617.

17 P. S. R. Prasad, K. Shiva Prasad and N. K. Thakur, Spectrochim. Acta. A. Mol. Biomol. Spectrosc., 2007, 68, 1096-1100.

18 J. E. Bertie and D. A. Othen, Can. J. Chem., 1973, 51, 1159-1168.

19 J. E. Bertie and D. A. Othen, Can. J. Chem., 1972, 50, 3443-3449.

20 F. Fleyfel and J. P. Devlin, J. Phys. Chem., 1991, 95, 3811-3815.

21 C. Lo, J. Zhang, P. Somasundaran and J. W. Lee, J. Colloid Interface Sci., 2012, 376, 173-176.

22 Y. Jin, H. Oyama and J. Nagao, Jpn. J. Appl. Phys., 2009, 48, 108001.

23 I. A. Monreal, L. Cwiklik, B. Jagoda-Cwiklik and J. P. Devlin, J. Phys. Chem. Lett., 2010, 1, 290-294.

24 N. Uras-Aytemiz, F. M. Balcı, Z. Maşlakcı, H. Özsoy and J. P. Devlin, J. Phys. Chem. A, 2015, 119, 9018-9026.

25 P. S. R. Prasad, K. Shiva Prasad and N. K. Thakur, Curr. Sci., 2006, 90, 1544-1547.

26 G. T. Dobbs, Y. Luzinova, B. Mizaikoff, Y. Raichlin and A. Katzir, Proceedings of the 6th International Conference on Gas Hydrates (ICGH 2008), Vancouver, 2008.

27 Y. Luzinova, G. T. Dobbs, Y. Raichlin, A. Katzir and B. Mizaikoff, Chem. Eng. Sci., 2011, 66, 5497-5503.

28 M. Schwenk, A. Katzir and B. Mizaikoff, Anal Methods, 2016, 8, 5897-5905.

29 N. J. Harrick, Internal Reflection Spectroscopy, Interscience, New York, 1967.

30 B. Mizaikoff, Meas. Sci. Technol., 1999, 10, 1185-1194.

31 J.-B. Brubach, A. Mermet, A. Filabozzi, A. Gerschel and P. Roy, J. Chem. Phys., 2005, 122, 184509 (1-7).

32 A. Millo, Y. Raichlin and A. Katzir, Appl. Spectrosc., 2005, 59, 460-466. 
1

2

3

4

5

6

7

8

9

10

11

12

13

14

15

16

17

18

19

20

21

22

23

24

25

26

27

28

29

30

31

32

33

34

35

36

37

38

39

40

41

42

43

44

45

46

47

48

49

50

51

52

53

54

55

56

57

58

59

60

33 K. Mizuno, Y. Masuda, T. Yamamura, J. Kitamura, H. Ogata, I. Bako, Y. Tamai and T. Yagasaki, J. Phys. Chem. B, 2009, 113, 906915.

34 H. H. Richardson, P. J. Wooldridge and J. P. Devlin, J. Chem. Phys., 1985, 83, 4387.

35 G. T. Dobbs, Ph. D. Thesis, Georgia Institute of Technology, 2007. 